\title{
LETTER
}

\section{Electrical impedance measurement of hydrous microcrystalline quartz}

\author{
Junichi FuKUdA ${ }^{*}$, Colin J. Peach ${ }^{* *}$, Christopher J. SPIERs ${ }^{* *}$ and Satoru NAKASHIMA* \\ *Department of Earth and Space Science, Graduate School of Science, Osaka University \\ 1-1 Machikaneyama, Toyonaka, Osaka 560-0043, Japan \\ **High Pressure and Temperature Laboratory, Department of Earth Sciences, Utrecht University, \\ P.O. Box 80.021, 3508 TA Utrecht, The Netherlands
}

\begin{abstract}
High temperature impedance spectra, up to $450{ }^{\circ} \mathrm{C}$, were measured for a microcrystalline quartz aggregate (chalcedony), which initially contained $0.3 \mathrm{wt} \%$ of liquid-like water $\left(\mathrm{H}_{2} \mathrm{O}\right)$ dispersed in grain boundaries and fluid inclusions together with $0.3 \mathrm{wt} \%$ of hydroxyl $(\mathrm{Si}-\mathrm{OH})$ in the crystal structure. Infrared spectra obtained after heating, showed dominant dehydration of liquid-like water, while much hydroxyl remained stable. Electrical conductivities before (wet) and after heating to $450{ }^{\circ} \mathrm{C}$ (dry) gave linear Arrhenius relations with apparent activation energies of $11 \pm 1 \mathrm{~kJ} / \mathrm{mol}$ for initial heating of the wet sample versus $32 \pm 3 \mathrm{~kJ} / \mathrm{mol}$ for the subsequently dry sample. Compared with previously reported Arrhenius relations for $\alpha$-quartz single crystals, our activation energies are much lower, and the absolute conductivities we obtained range from similar values to three orders of magnitude higher. We infer that the presence of grain boundaries and/or triple junctions containing liquidlike water greatly influences the electrical conductivity.
\end{abstract}

Keywords: Liquid-like water, Microcrystalline quartz, Electrical conductivity, Dehydration, Chalcedony, Impedance spectroscopy

\section{INTRODUCTION}

It is well known that natural rocks contain molecular water confined in grain boundaries, triple junctions and fluid inclusions (hereafter referred to as liquid-like water; Nakashima et al. 2004). Liquid-like water has significant influences on the rheology of the Earth's crust through deformation processes such as pressure solution, fluidassisted recrystallization, grain growth, and fluid-assisted cataclasis, as summarized in Dysthe and Wogelius (2006). Of key importance in controlling these processes and the associated rheology is the distribution and properties of the liquid-like water phase. Electrical conductivity is a useful tool for evaluating the distribution and properties of liquid-like water in rocks, since it is highly sensitive to conduction through high diffusivity, fluid-filled pathways (including hydrous fluids and melts).

Numerous data on electrical conductivity have been

doi:10.2465/jmps.081022f

J.Fukuda, jfukuda@ess.sci.osaka-u.ac.jp Corresponding author

C.J.Peach, cpeach@geo.uu.nl

C.J.Spiers, cspiers@geo.uu.nl

S. Nakashima, satoru@ess.sci.osaka-u.ac.jp reported for various materials, including single crystals, glass and grain aggregates, many of which are summarized in Watanabe (2005). Recently, electrical conductivity has been measured for natural crustal rocks dependent on rock texture, under high pressure-temperature conditions (e.g., Fuji-ta et al., 2004; Fuji-ta et al., 2007). Also, several attempts to evaluate the effects of liquid-like water on electrical conductivities have been reported for a few simple granular aggregates (e.g., Watanabe and Peach, 2002 for salt rock; Wang et al., 2006 for olivine aggregates) and for bi-crystal contacts of salt rock undergoing pressure solution (De Meer et al., 2005; Van Noort et al., 2007). However, materials used in experiments and data are thus limited and the influence of liquid-like water on electrical conductivities in low permeability crystalline rock and fine grained microporous material is poorly understood.

We report the changes of electrical conductivity associated with dehydration of liquid-like water in microcrystalline quartz, viewing it as an analogue for quartzrich rocks in the Earth's crust. Electrical impedance spectra were continuously measured at several high temperatures up to $450{ }^{\circ} \mathrm{C}$. The difference in electrical con- 

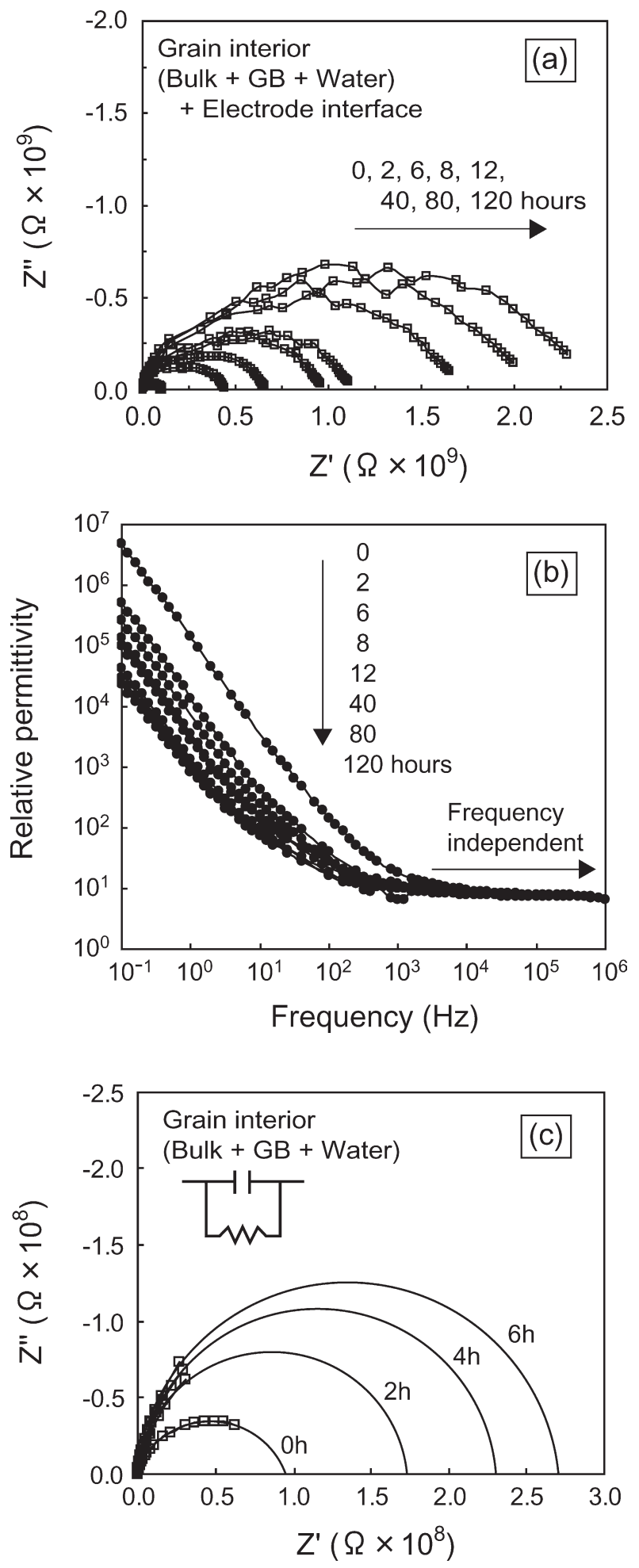

ductivity between initially wet and subsequently dried samples is also addressed and an attempt is made to understand the distribution of water in the samples.

\section{MATERIALS AND EXPERIMENTAL MEHODS}

Microcrystalline chalcedonic quartz samples were collected from Chayagawa, Oshamanbe, South-West Hokkaido, Japan. The water content and stability at high temperature of such a sample was roughly determined by insitu infrared (IR) spectroscopy in our previous study, which demonstrates that liquid-like water gradually dehydrates at less than $400{ }^{\circ} \mathrm{C}$ (Fukuda and Nakashima, 2008). Disc-shaped samples of $13 \mathrm{~mm}$ diameter and between 1.1-1.2 mm thick were used for the impedance measurement. To ensure good electrical contact, disc-shaped electrodes of 8 and $12 \mathrm{~mm}$ diameter were deposited onto the two opposite faces of the samples by gold sputtering in a partial vacuum. A guard ring of $1 \mathrm{~mm}$ was also sputtered around the $8 \mathrm{~mm}$ electrode to ensure conduction directly through the sample.

The complex impedance spectra were measured using a Solartron 1260A frequency response analyzer with a Solartron 1296A dielectric interface for an increased impedance range up to $10^{14} \Omega$ and capacitance down to $10^{-12}$ F. Systematic measurement errors with the combined apparatus are less than $2 \%$. The conductivity cell, constructed of refractory alumina (Ten Grotenhuis et al., 2004), was equipped with $0.5 \mathrm{~mm}$ thick Pt electrodes and guard ring, of similar areal dimensions to the sputtered sample electrodes, to ensure good connection via shielded platinum wires. This was situated inside a $60 \mathrm{~mm}$ internal diameter tube furnace, with temperature controlled and monitored using $\mathrm{Pt} / \mathrm{Pt}-10 \% \mathrm{Rh}$ thermocouples. The real $Z^{\prime}$ and imaginary $Z^{\prime \prime}$ components of the impedance were obtained in the frequency range of $0.1 \mathrm{~Hz}$ to $1 \mathrm{MHz}$ with a periodic sinusoidal r.m.s. voltage of $200 \mathrm{mV}$. The complex impedance spectra were continuously measured at constant temperatures of 250,350 , and $450{ }^{\circ} \mathrm{C}$ under open

Figure 1. (a) Complex impedance spectra series; an example of continuous heating at $350{ }^{\circ} \mathrm{C}$. The changes of arcs are shown by time-line. One single arc grows less circular and larger with exposure time. These arcs contain contributions from sample interior (bulk and grain boundaries (GB in the figure) or triple junctions/inclusions containing liquid-like water) and predominant charge-up of the electrodes (seen at lower frequencies; most of visible data points are $<1 \mathrm{kHz}$ ). Changes in arc diameter above 1 $\mathrm{kHz}$ are due solely to dehydration whilst those at lower frequencies include charging of the electrodes. This influence becomes larger with exposure (heating) time, excessively stretching the arcs towards larger apparent real impedances (resistances). (b) Changes of corresponding relative permittivities. Permittivities are frequency independent above $1 \mathrm{kHz}$, but grow rapidly with decrease of frequency below that value (phenomenon of low frequency dispersion; possible ionic-charge buildup on electrodes in layers with associated high capacitance). (c) Some fitted arcs to simple RC circuit below $1 \mathrm{kHz}$ (30 data points per arc). 


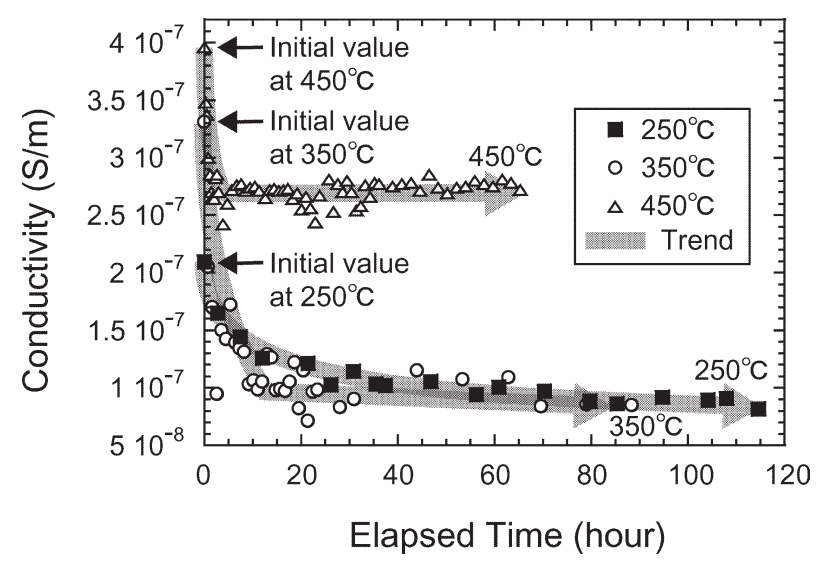

Figure 2. Changes of electrical conductivities against exposure time to heating at 250,350 and $450{ }^{\circ} \mathrm{C}$. Trends of decreasing conductivities are also shown in gray arrows. The decreases in conductivities are due to the dehydration of liquid-like water.

atmosphere conditions for periods up to 120 hours, and spectral changes associated with dehydration were obtained as a function of time.

Before and after the experiments, the liquid-like water $\left(\mathrm{H}_{2} \mathrm{O}\right)$ and hydroxyl $(\mathrm{Si}-\mathrm{OH})$ contents were separately determined using IR spectroscopy (Jasco FT-IR $470+$ IRT-30 instruments). The IR apparatus is equipped with a Glober (silicon carbide) IR source, a Ge-coated $\mathrm{KBr}$ beamsplitter, and a mercury-cadmium-telluride detector. The spectra were obtained by multiple scanning (256 times) with a resolution of $4 \mathrm{~cm}^{-1}$.

\section{RESULTS AND DISCUSSION}

Changes of complex impedance spectra and relative permittivity spectra at $350{ }^{\circ} \mathrm{C}$ are shown in Figures $1 \mathrm{a}$ and $1 \mathrm{~b}$, respectively, as an example. Roughly, a single arc grows larger with increased exposure time to heating. Since the solid-state conductivity of dry quartz is very low at these conditions (Jain and Nowick, 1982), conductivity is expected to be dominated by other, mainly intercrystalline pathways. The arcs can be considered to be due to the behavior of the sample interior and sample electrode interface effects. The sample interior components will contain contributions from bulk quartz, and its grain boundaries, triple junctions or inclusions containing liquid-like water. Electrode interface effects will be related to charge transfer (from/to ions in solution to/from electrons in metal connectors, as redox reactions) and capacitive storage charge/discharge (e.g., via Gouy-Chapman double-layers). At low frequencies, electrode interface effects are confirmed by the presence of low frequency dispersion or LFD (Jonscher, 1991), seen as a significant increase in cell-capacitance towards lower fre- quencies. This is most likely due to the charge-up of the electrode by accumulation of transported ions in doublelayer structures. Above $1 \mathrm{kHz}$, the cell capacitance (that corresponds to cell permittivity) is frequency independent (Fig. 1b) and so only this part of the arcs was used to estimate the conduction related impedances from a simple parallel RC circuit model, where capacitance $\mathrm{C}$, must be assumed constant to extract the resistance $\mathrm{R}$, related to ionic transport, from the arc diameter. The size of the arcs grows with continued dehydration as can be seen by measuring continuously (Fig. 1a), but the high frequency changes in arc diameter are less than this figure suggests since the majority of the visible data is for frequencies below $1 \mathrm{kHz}$ and is also affected by a frequency dependent growth in capacitance and interface resistance (LFD). By only using the constant cell capacitance, LFD free data above $1 \mathrm{kHz}$, we avoid the complications of the electrode charging effects. The results of arc fitting are shown in Figure 1c. Electrical conductivity, $\sigma$ originating from the sample interior is calculated by $\sigma=\mathrm{d} / \mathrm{SZ}^{\prime}$, where $\mathrm{d}$ is the sample thickness, $\mathrm{S}$ is the measured area $(8 \mathrm{~mm}$ diameter electrode), and $Z^{\prime}$ is the real part of the impedance or the resistance, $\mathrm{R}$. This was determined from the intersection of the arc, projected from just the data segment above 1 $\mathrm{kHz}$, with the real impedance axis (pure resistance) by numerically fitting a simple parallel RC circuit model (Fig. 1c), as a best fit semicircle. Some fitted arcs are shown in Figure 1c. When the data above $1 \mathrm{kHz}$ is viewed more closely then there is no sign of additional arcs that could suggest any separate pathways or additional mechanisms for conduction, but the data do follow a more circular single arc, with very little depression, allowing a good fit to the simple parallel RC model. Changes of electrical conductivity at each temperature against the duration of heat treatment (heat exposure time) are shown in Figure 2.

IR spectral analysis shows the changes in water content before and after the experiment (Fig. 3). Water content, including liquid-like water $\left(\mathrm{H}_{2} \mathrm{O}\right)$ and hydroxyl $\left(\mathrm{Si}^{-}\right.$ $\mathrm{OH})$, can be calculated separately from each coupling vibration mode of stretching and bending, which can be recognized around 5200 and $4500 \mathrm{~cm}^{-1}$, respectively (Graetsch et al., 1985). This calculation gives the true water content to the first decimal place, but the relative value can be more closely compared within the same sample as Figure 3. Initially, water content, as we described, was $0.32 \mathrm{wt} \%$ of liquid-like water and $0.28 \mathrm{wt} \%$ of hydroxyl in Figure 3. After the experiment, dominant dehydration of liquid-like water is confirmed, while much hydroxyl remains stable. In the region of the water stretching band around $3800-3000 \mathrm{~cm}^{-1}$, a new peak at $3740 \mathrm{~cm}^{-1}$ can be recognized after the heating experiment, especially after exposure to higher temperatures. This peak is considered 

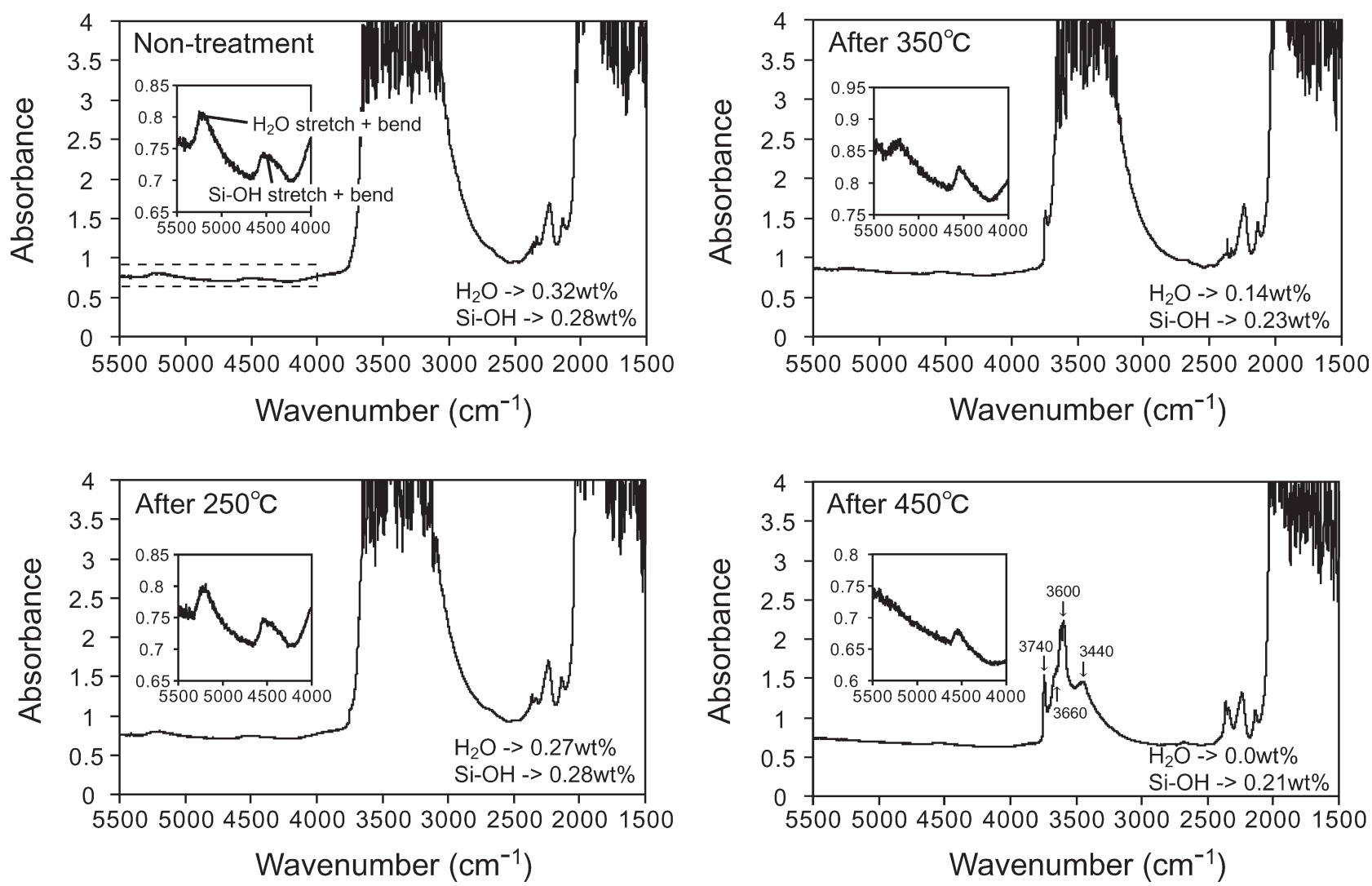

Figure 3. Infrared spectra before heating (initial) and after heating at 250,350 and $450{ }^{\circ} \mathrm{C}$. Liquid-like water $\left(\mathrm{H}_{2} \mathrm{O}\right)$ and hydroxyl $(\mathrm{Si}-\mathrm{OH})$ contents were calculated from each stretching and bending combination band around $5200 \mathrm{~cm}^{-1}$ and $4500 \mathrm{~cm}^{-1}$, respectively. To compare the relative value after heating, values are shown to the second decimal place (See the text for detailed explanation). A dominant decrease of liquidlike water can be seen after heating.

to be the isolated surface silanol, which is not hydrogen bonded with liquid-like water and appears after dehydration of liquid-like water starts (Yamagishi et al., 1997). According to the results of IR spectroscopy, the changes of resistance (Fig. 1) and conductivity (Fig. 2) with heat treatment duration or exposure time must mainly reflect the dehydration of liquid-like water.

In Figure 2, the initial values of conductivity can only reflect the temperature dependence of the hydrous samples, since liquid-like water trapped in grain boundaries or triple junctions, or structurally incorporated as hydroxyl, has not yet been driven out of the samples. The initial value at each temperature is $4.0 \times 10^{-7} \mathrm{~S} / \mathrm{m}$ at 450 ${ }^{\circ} \mathrm{C}, 3.3 \times 10^{-7} \mathrm{~S} / \mathrm{m}$ at $350{ }^{\circ} \mathrm{C}$, and $2.1 \times 10^{-7} \mathrm{~S} / \mathrm{m}$ at 250 ${ }^{\circ} \mathrm{C}$. Separate from the dehydration experiments at these three temperatures, initial conductivity at $200{ }^{\circ} \mathrm{C}$ for a fourth wet sample was determined and the value was 1.5 $\times 10^{-7} \mathrm{~S} / \mathrm{m}$.

With exposure to high temperatures, liquid-like water dominantly dehydrates as described above. In the case of $450^{\circ} \mathrm{C}$, conductivity quickly decreased from $4.0 \times 10^{-7}$ $\mathrm{S} / \mathrm{m}$ to an almost constant value (ca. $2.7 \times 10^{-7} \mathrm{~S} / \mathrm{m}$ ) from 5 hours onwards. Coupled with the result of IR spectros- copy (Fig. 3), these constant values correspond to the state at which the sample has no liquid-like water. Therefore, these values come from grain boundaries and quartz itself. At temperatures of 250 and $350{ }^{\circ} \mathrm{C}$, although both conductivities reached similar values during the experimental heat treatment, the conductivities obtained depend on both the water contents and temperatures. That is specifically considered as follows. Calculated water contents after the experiments show dominant and different degree of dehydration of liquid-like water depending on heating temperatures; 0.14 and $0.27 \mathrm{wt} \%$ for after $350{ }^{\circ} \mathrm{C}$ and 250 ${ }^{\circ} \mathrm{C}$, respectively (Fig. 3). These differences of water contents have influences on values of electrical conductivities. On the other hand, the conductivity increases with temperature increase even in the same sample composition. The combination of water content and temperature reflect the obtained similar value of conductivities at 350 and $250^{\circ} \mathrm{C}$.

After the experiment at $450{ }^{\circ} \mathrm{C}$, i.e., for the dry sample, conductivity was again measured at several temperatures down to $100{ }^{\circ} \mathrm{C}$. These values together with the initial values (i.e., wet) for the other samples at each temperature described above were plotted in an Arrhenius 


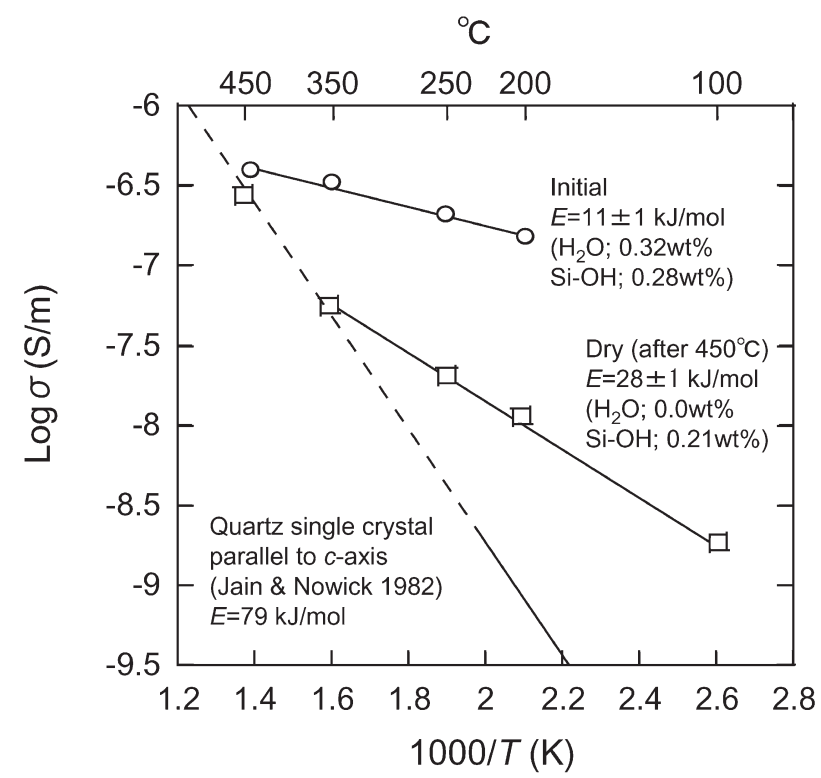

Figure 4. Arrhenius diagram of electrical conductivity $(\mathrm{S} / \mathrm{m})$ for before (initial) and after prolonged heating (dry) at $450{ }^{\circ} \mathrm{C}$. Water contents in the form of liquid-like water $\left(\mathrm{H}_{2} \mathrm{O}\right)$ and hydroxyl $\left(\mathrm{Si}^{-}\right.$ $\mathrm{OH})$ are also shown. Conductivity becomes lower and activation energy becomes higher for the dry sample, indicating the additional conduction due to liquid-like water in the wet (initial) sample. The typical value for a natural $\alpha$-quartz single crystal parallel to crystallographic $\boldsymbol{c}$-axis reported by Jain and Nowick (1982) is also shown as solid line for their measured data and dotted line for extrapolated data.

diagram (Fig. 4). Apparent activation energies for the dry and wet sample were determined from the Arrhenius relation; $\sigma=\sigma_{0} \exp (-E / R T)$, where $\sigma$ is the conductivity, $\sigma_{0}$ is the conductivity at infinitely high temperature, $E$ is the apparent activation energy, $R$ is the molar gas constant, and $T$ is absolute temperature. The data for the dry sample were fitted excluding that of $450{ }^{\circ} \mathrm{C}$, since the data at 450 ${ }^{\circ} \mathrm{C}$ seems to deviate from other four temperature data and may represent a conduction process more intrinsic to the bulk quartz rather than conduction along grain boundaries or triple junction tubes. This calculation gives the apparent activation energies and pre-exponential factors $\sigma_{0}$ of respectively $11 \pm 1 \mathrm{~kJ} / \mathrm{mol}$ and $0.28 \times 10^{-5}$ for the wet sample, versus $32 \pm 3 \mathrm{~kJ} / \mathrm{mol}$ and $0.38 \times 10^{-4}$ for the dry sample. Good linearity can be seen. However, water on the saturation liquid vapor curve is known to exhibit a maximum conductivity at around $230{ }^{\circ} \mathrm{C}$ (IAPWS, 1990) and therefore cannot produce a linear Arrhenius plot. This means that the apparent activation energy for wet samples probably contains contributions due to variations in distribution, connectivity and fluid density. The Arrhenius relations obtained show a large difference from those for single quartz crystals. According to the determination by Jain and Nowick (1982), for a natural $\alpha$-quartz single crystal parallel to crystallographic $c$-axis (their trend is also shown in Fig. 4), the activation energies in their report is $79 \mathrm{~kJ} / \mathrm{mol}$ which are much higher values than determined in our experiments. Moreover, conductivities obtained in our study have a similar value ranging to three orders of magnitude higher in our experimental range. The quartz single crystal data shows that electrical conduction of quartz itself becomes dominant over $450{ }^{\circ} \mathrm{C}$ because of higher activation energy. Therefore, the deviation of our dry sample at $450{ }^{\circ} \mathrm{C}$ is best explained by a change of charge carrier over $450{ }^{\circ} \mathrm{C}$ from water in grain boundaries to the ionic conduction within quartz itself as given by the Jain and Nowick (1982) data trend in Figure 4.

The distribution of liquid-like water can be roughly evaluated. We consider here the water distribution at 350 ${ }^{\circ} \mathrm{C}$, for example, using reported conductivity data for pure water from IAPWS (1990). The liquid-like water content of $0.3 \mathrm{wt} \%$ translates to $0.77 \mathrm{vol} \%$. The grain size must correspond to the crystallite size of $30 \mathrm{~nm}$ (Fukuda and Nakashima, 2008). If the fluid is distributed evenly on grain faces then the water content represents a layer 0.1 $\mathrm{nm}$ thick, which is only half a monolayer of water molecules $(\mathrm{O}-\mathrm{H}$ bond length is approximately $0.096 \mathrm{~nm})$. In this restricted state, bound between adjacent grains, water is not likely to behave as liquid water. However, water is observed to be present in a liquid-like state, (Fukuda and Nakashima, 2008; as inferred from in-situ IR spectroscopy) so for the thickness of boundary water to be great enough to allow such freedom to molecules then it must be partially covering ( $<50 \%$ of grain faces) and possibly disconnected. Hence, a distribution in triple junction tubes is more likely. Therefore, we assume that liquid-like water is evenly distributed in fully-connected grain-edge tubes. Taking the electrical conductivity of pure water as $6.0 \times 10^{-5} \mathrm{~S} / \mathrm{m}$ at $350{ }^{\circ} \mathrm{C}$ (fluid density $=0.7 \mathrm{~g} / \mathrm{cm}^{3}$ ), a connected grain-edge tube model (Grant and West, 1965; Schmeling, 1986; Ten Grotenhuis et al., 2005) then gives the calculated conductivity as $2.7 \times 10^{-6} \mathrm{~S} / \mathrm{m}$, which is about one order of magnitude higher than the experimentally determined value of $3.3 \times 10^{-7} \mathrm{~S} / \mathrm{m}$. Conductivity of pure water has a maximum at $230{ }^{\circ} \mathrm{C}$, a factor of $\sim 15$ higher than at $350{ }^{\circ} \mathrm{C}$, as described above. So, the fullyconnected grain-edge model overestimates the bulk conductivity by more than 2 orders at the lower temperatures studied. Therefore, we infer that liquid-like water in tubes must either expand, (limited by thickness restrictions argued above), to fill grain boundaries lowering its density and hence conductivity at high temperatures or it becomes lost during heating prior to measurement, or the fluidfilled tubes are partially disconnected before or during heating.

We conclude from our results that below $350{ }^{\circ} \mathrm{C}$ it is the presence of grain boundaries and of liquid-like water 
within triple junction tubes, or penetrating grain boundaries on heating, that leads to the high electrical conduction seen in comparison with quartz single crystals.

\section{ACKNOWLEDGEMENTS}

The authors thank P. van Krieken, G. Kastelein and E. de Graaff for their technical supports of sample preparation and apparatuses operation. Two anonymous reviewers are also thanked for their constructive comments as well as A. Yoshiasa for editorial handling. This work was financially supported by "Collaboration Project with the EU Erasmus Mundus Program for Internationalized Education and Research of Basic Science" under an international education project "International Training Program" run by the Independent Administrative Agency, Japan Society for the Promotion of Science.

\section{REFERENCES}

De Meer, S., Spiers, C.J. and Nakashima, S. (2005) Structure and diffusive properties of fluid-filled grain boundaries: An in-situ study using infrared (micro) spectroscopy. Earth and Planetary Science Letters, 232, 403-414.

Dysthe, D.K. and Wogelius, R.A. (2006) Confined fluids in the Earth's crust - Properties and processes. Chemical Geology, 230, 175-181.

Fukuda, J. and Nakashima, S. (2008) Water at high temperatures in a microcrystalline silica (chalcedony) by in-situ infrared spectroscopy: physicochemical states and dehydration behavior. Journal of Mineralogical and Petrological Sciences, 103, $112-115$.

Fuji-ta, K., Katsura, T. and Tainosho, Y. (2004) Electrical conductivity measurement of granulite under mid- to lower crustal pressure-temperature conditions. Geophysical Journal International, $157,79-86$.

Fuji-ta, K., Katsura, T., Matsuzaki, T., Masahiro, I. and Kobayashi, T. (2007) Electrical conductivity measurement of gneiss under mid- to lower crustal P-T conditions. Tectonophysics, 434, 93-101.

Graetsch, H., Flörke, O.W. and Miehe, G. (1985) The nature of water in chalcedony and opal-C from Brazilian agate geodes. Physics and Chemistry of Minerals, 12, 300-306.

Grant, F.S. and West, G.F. (1965) Introduction to electrical methods. In Interpretation theory in Applied Geophysics(Shrock,
R.R. Ed.). McGraw-Hill, New York, 385-401.

IAPWS guideline statement (1990) Electrolytic conductivity (specific conductance) of liquid and dense supercritical water from $0{ }^{\circ} \mathrm{C}$ to $800{ }^{\circ} \mathrm{C}$ and pressure up to $1000 \mathrm{MPa}$. http:// www.iapws.org/relguide/conduct.pdf.

Jain, H. and Nowick, A.S. (1982) Electrical conductivity of synthetic and natural quartz crystals. Journal of Applied Physics, 53, 477-484.

Jonscher, A.K. (1991) Low-frequency dispersion in volume and interfacial situations. Journal of Materials Science, 26, 16181626.

Nakashima, S., De Meer, S. and Spiers, C.J. (2004) Distribution of thin film water in grain boundaries of crustal rocks and implications for crustal strength. In Physiochemistry of Water in Geological and Biogeological Systems - Structures and Properties of Thin Aqueous Films (Nakashima, S., Spiers, C.J., Mercury, L., Fenter, P.A. and Hochella Jr., M.F., Eds). Frontier Science Series, 44, Universal Academy Press, Tokyo. 159-178.

Van Noort, R., Spiers, C.J. and Peach, C.J. (2007) Effects of orientation on the diffusive properties of fluid-filled grain boundaries during pressure solution. Physics and Chemistry of Minerals, 34, 95-112.

Schmeling, H. (1986) Numerical models on the influence of partial melt on elastic, anelastic and electrical properties of rocks. Part II: Electrical conductivity. Physics of the Earth and Planetary Interiors, 43, 123-136.

Ten Grotenhuis, S.M., Drury, M.R., Peach, C.J. and Spiers, C.J. (2004) Electrical properties of fine-grained olivine: Evidence for grain boundary transport. Journal of Geophysical Research, 109 (B6), B0623, doi:10.1029/2003JB002799.

Ten Grotenhuis, S.M., Drury, M.R., Spiers, C.J. and Peach, C.J. (2005) Melt distribution in olivine rocks based on electrical conductivity measurements. Journal of Geophysical Research, 110, B12201, doi:10.1029/2004JB003462.

Wang, D. and Mookherjee, M., Xu, Y., Karato, S. (2006) The effect of water on the electrical conductivity of olivine. Nature, 443, 977-980.

Watanabe, T. (2005) Electrical Properties of Rocks: A Review. Journal of Geography, 114, 837-861 (in Japanese with English abstract).

Yamagishi, H., Nakashima, S. and Ito, Y. (1997) High temperature infrared spectra of hydrous microcrystalline quartz. Physics and Chemistry of Minerals, 24, 66-74.

Manuscript received October 22, 2008

Manuscript accepted January 23, 2009

Published online April 17, 2009

Manuscript handled by Akira Yoshiasa 